\title{
Immunohistochemistry in apparently normal bone marrow trephine specimens from patients with nodal follicular lymphoma
}

\author{
R Chetty, G Echezarreta, M Comley, K Gatter
}

\begin{abstract}
Aim-To establish the role of immunohistochemistry (using a limited panel of antibodies) in detecting minimal involvement by follicular lymphoma in routinely processed bone marrow trephine specimens, which show no obvious morphological (light microscopic) evidence of lymphoma; to determine whether bcl-2 immunostaining in bone marrow distinguishes between benign and malignant infiltrates in a patient with nodal follicular lymphoma.
\end{abstract}

Methods-Twenty seven consecutively selected paraffin wax embedded, formalin fixed bone marrow trephine specimens were stained with the following antibodies: anti-bcl-2, anti-CD79a, anti-CD3, and $\kappa$ and $\lambda$ light chains, using the Streptavidin biotin complex technique.

Results-Five of the 27 cases, which showed no evidence of involvement by follicular lymphoma on routine stains, showed monotypic $B$ cells on immunohistochemistry. Two of the cases were diffuse, while the remaining three showed mini-aggregates around bony trabeculae. In all five cases the lymphomatous infiltrates were strongly bcl-2 positive. Reactive $B$ lymphoid nodules did not show the same degree of bcl-2 positivity, and negative cells could be discerned within the reactive nodules.

Conclusions-There is merit in studying so-called negative bone marrows immunohistochemically in order to detect minimal involvement by follicular lymphoma. A limited panel of antibodies including anti-bcl-2, anti-CD79a and antiCD3 is usually adequate to accomplish this. Strongly bcl-2 positive lymphoid aggregates in the bone marrow of patients with nodal follicular lymphoma are indicative of lymphoma.

(f Clin Pathol 1995;48:1035-1038)

Keywords: Immunohistochemistry, bone marrow trephine specimens, nodal follicular lymphoma.

University

Department of

Cellular Science, Level 4,

Academic Block,

John Radcliffe

Hospital,

Oxford OX3 9DU

Correspondence to: Dr R Chetty.

Accepted for publication 1 June 1995

Once a diagnosis of lymph node based follicular lymphoma is made, a bone marrow trephine biopsy is usually performed as part of the staging process. Obvious involvement of bone marrow by follicular lymphoma is characteristically para-trabecular in distribution and is easily detected using routine haema- toxylin and eosin and Giemsa stains. However, subtle infiltrates are usually obscured by other marrow cells and consequently are not easily seen. These trephine specimens are frequently reported as normal or as no obvious infiltrate being present.

In this study cases of nodal follicular lymphomas lacking obvious bone marrow involvement were examined using a limited panel of antibodies. The aim was to determine whether early or minimal lymphomatous involvement could be detected and reliably distinguished from benign reactive lymphoid infiltrates.

\section{Methods}

Twenty seven consecutive cases of follicular lymphoma and their corresponding formalin fixed, paraffin wax embedded bone marrow trephine specimens, which were initially reported as being negative for lymphoma, were examined. The diagnosis of follicular lymphoma was confirmed by re-examination of the light microscopic and immunohistochemical findings. The bone marrow trephine biopsies in these cases were performed as part of a routine staging procedure. Initially, the bone marrow trephine specimens were stained only with haematoxylin and eosin or Giemsa stains, or both. For this study the bone marrow trephine specimens were stained with JCB 117 (CD79a, neat), anti-bcl-2 (BCL-2 124, neat) and anti-CD3 (polyclonal, diluted 1 in 100), all from Dako, High Wycombe, UK. Staining for light chain $(\kappa$ and $\lambda$ ) restriction was also carried out.

The Streptavidin biotin complex technique with 3,3'-diaminobenzidine as chromagen was used. Slides were heated in a microwave oven prior to staining.

Twenty five reactive/normal bone marrow trephine specimens from patients with no history of leukaemia, lymphoma or other disease likely to cause lymphoid aggregates in the bone marrow, were stained in parallel. Ten cases of known follicular lymphoma involving the bone marrow were similarly stained.

\section{Results}

REACTIVE/NORMAL BONE MARROW TREPHINE SPECIMENS

$\mathrm{T}$ and B lymphocytes were scattered randomly in a non-specific, interstitial pattern. No clustering around bony trabeculae was noted. Occasional aggregates of $\mathrm{T}$ and polyclonal $\mathrm{B}$ cells 


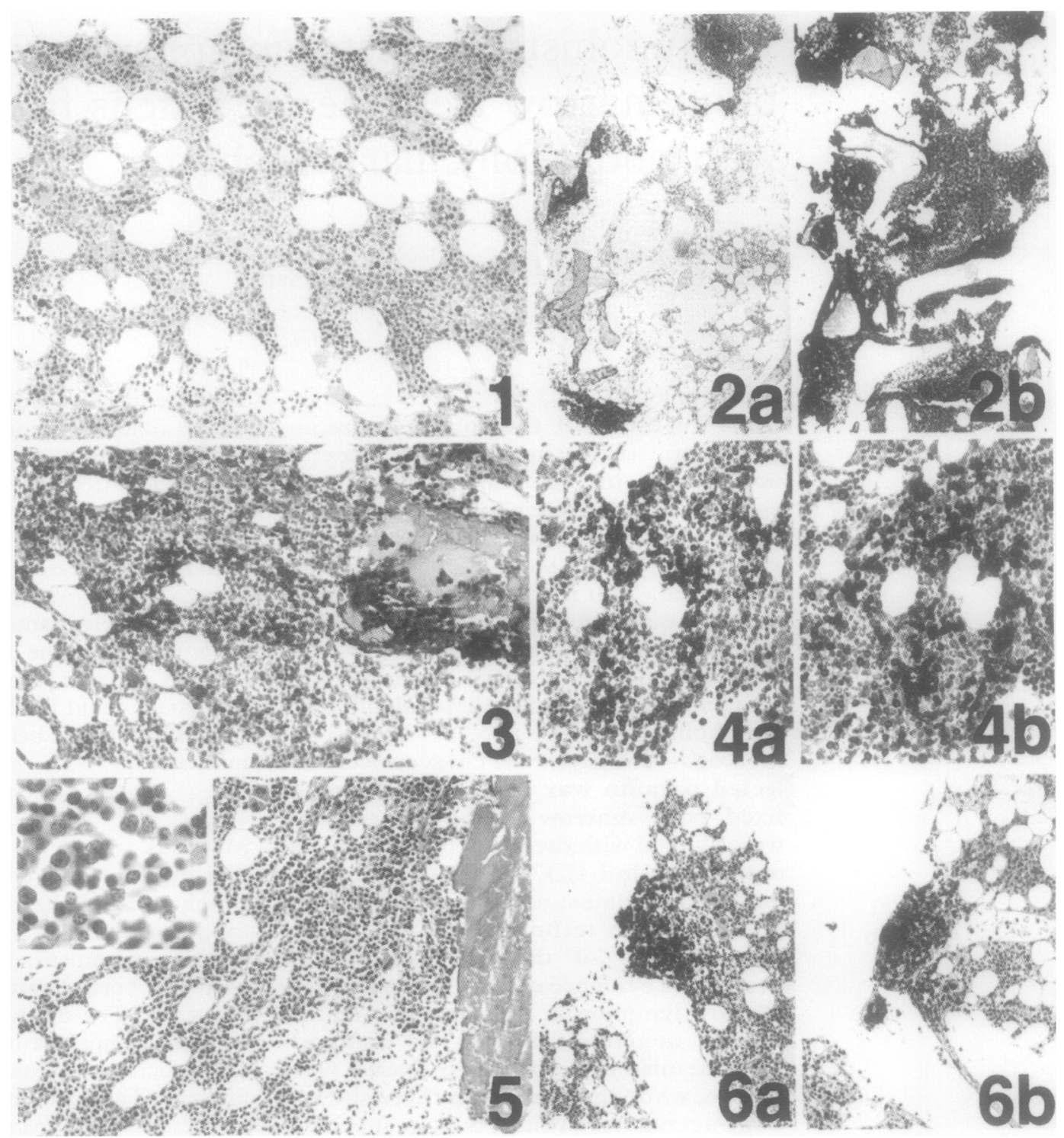

Figure 1 Staining of a normal/reactive bone marrow with anti-CD79a. Very occasional, scattered B cells are present. No clustering or mini-aggregates of lymphoid cells are evident. B cells account for approximately $5 \%$ of the cells in the reactive marrow.

Figure 2 Obvious follicular lymphoma in the marrow stained with anti-CD79a (2a). The typical para-trabecular distribution is present. A heavier infiltrate of follicular lymphoma stained with anti-bcl-2 (2b).

Figure $3 A$ case of follicular lymphoma stained with anti-CD79a showing an interstitial or dispersed pattern of involvement. A greater number of $B$ cells is present compared with the reactive trephine specimen in fig 1 . The increase in $B$ cell numbers can only be appreciated after immunohistochemical staining.

Figure 4 Higher power photomicrograph of the dispersed pattern of lymphomatous marrow infiltration stained with anti$C D 79 a(4 a)$ and the same area with anti-bcl-2 (4b). The slightly increased number of cells stained with anti-bcl-2 is because of the presence of occasional T lymphocytes.

Figure 5 Routine haematoxylin and eosin stained section of a bone marrow trephine specimen containing minimal follicular lymphoma with a dispersed pattern. There is some hypercellularity but unequivocal comment cannot be made as to the nature of the infiltrate. The inset shows some of the atypical lymphoid cells admixed with normal marrow elements. Figure 6 Micro-aggregates of minimal follicular lymphoma in the marrow. Staining with anti-CD79a (6a) and antibcl-2 (6b) once again highlights the extent and aggregation of the infiltrate. These clusters were not apparent on the haematoxylin and eosin stained section.

were present. The reactive $B$ cell aggregates contained occasional CD79a positive cells (fig 1). In these reactive/normal marrows, mature T lymphocytes outnumbered mature $B$ cells by about $2: 1$. The overall percentage of $T$ and $B$ cells relative to the other marrow elements was about $10-15 \%$ and $5 \%$, respectively.

BONE MARROW TREPHINE SPECIMENS IN PATIENTS WITH NODAL FOLLICULAR LYMPHOMA Obvious involvement

The 10 cases of obvious follicular lymphoma showed the typical pattern of involvement of the marrow. Immunohistochemistry with CD79a and bcl-2 (positive in all 10 cases) highlighted the pattern and extent of infiltration (figs $2 a$ and $2 b$ ).

No obvious marrow involvement on routine stains

Twenty two cases showed an identical pattern to the reactive/normal bone marrow trephine specimens. In the remaining five cases, distinct monotypic B cells were noted on immunohistochemistry. Interestingly, not all of the aggregates were para-trabecular in distribution or 
arranged in clusters. Two cases had a dispersed or interstitial pattern of involvement (fig 3). The extent of involvement once again was readily enhanced by the use of CD79a and bcl-2 (figs $4 \mathrm{a}$ and $4 \mathrm{~b}$ ). Re-evaluation of the routine haematoxylin and eosin stained sections did not reveal an obvious infiltrate (fig 5). A slight hypercellularity (with occasional atypical cells) was noted but was insufficient to diagnose lymphomatous infiltration confidently.

Clusters (seen in three of the cases) consisted of groups of cells staining strongly with CD79a and anti-bcl-2 (figs $6 a$ and $6 \mathrm{~b}$ ). The numbers of lymphocytes constituting the collections ranged from 25 to 50 cells.

A diffuse pattern was seen in two cases, where the B cell population accounted for more than the usual $5 \%$ of the cellular components (fig 3). These malignant cells were interspersed amongst the normal marrow elements, and the striking increase in their numbers was only evident on immunohistochemistry. Monoclonality of these infiltrates was not established unequivocally by seeking light chain restriction because background staining led to difficulty in interpreting these stains. However, the infiltrates in the five cases were strongly bcl-2 positive.

$\mathrm{T}$ and $\mathrm{B}$ cells both stained with anti-bcl-2. Plasma cells were also stained with this antibody, accentuating their perivascular distribution. CD79a also stained plasma cells strongly.

Clinically, nine of the 10 patients with obvious bone marrow involvement did not show any haematological abnormality. The remaining case had almost total effacement of the marrow with resultant symptoms of anaemia and thrombocytopenia. All 27 patients without obvious bone marrow disease did not have any specific symptoms suggestive of possible marrow involvement. The bone marrow trephine biopsies were carried out as part of a staging process. The five patients in whom follicular lymphoma of the marrow was detected by immunohistochemistry were asymptomatic and did not have any haematological abnormality. In this regard they did not differ from the 22 negative cases.

\section{Discussion}

The diagnostic usefulness of anti-CD79a, antibcl-2 and anti-CD3 antibodies on paraffin wax embedded material is well established. In fact, these three antibodies are part of a routine panel of diagnostic antibodies in many laboratories. In this paper we have highlighted their use in the detection of minimal marrow involvement by lymphomatous infiltrates. The diagnostic role of immunohistochemistry in the examination of bone marrow trephine specimens has been investigated previously. ${ }^{1}$ The cellular preservation and detail in bone marrow trephine specimens is often compromised by fixation regimens and decalcification procedures. Also, the presence of bony tissue, marrow and stroma render the bone marrow trephine specimen a technically difficult biopsy specimen on which to obtain quality sections routinely. Therefore, appreciation of subtle differences between the various cell types, and distinction and recognition of minimal infiltrates, is difficult.

The distribution of $\mathrm{T}$ and $\mathrm{B}$ cells in reactive/ normal bone marrow and their staining patterns with these three antibodies, forms an important basis on which lymphoid infiltrates can then be assessed. While compartmentalisation of $T$ and $B$ cells is characteristic of lymph nodes and extranodal lymphoid tissues, these cells are scattered randomly (a pattern described as "non-specific interstitial" by Shin et $a l$ ) or arranged as small non-trabecular aggregates in the bone marrow. $\mathrm{T}$ and $\mathrm{B}$ cells are thought to account for about $20 \%$ and $5 \%$ of the normal bone marrow, respectively. ${ }^{23}$ Non-lymphomatous infiltrates within bone marrow have two patterns: lymphoid nodules which resemble the Malpighian follicles of the spleen; and lymphoid infiltrates. ${ }^{4}$ The nodules tended to be within marrow fat and are not paratrabecular. They were made up of a polymorphous collection of cells (lymphocytes, and plasma, mast and reticulum cells). ${ }^{4}$ This contrasts with lymphomatous nodules, which generally contain one cell type only and are characteristically para-trabecular.

Lymphomatous infiltration of the bone marrow can result in several patterns: nodular, diffuse/interstitial, para-trabecular, and socalled packed marrow. ${ }^{15}$ Small infiltrates are difficult to diagnose on morphological grounds alone. ${ }^{4}$ Obvious infiltration is readily highlighted with the use of appropriate immunohistochemistry, and it is recommended that this is performed to characterise the nature of the infiltrate (for example, separation of lymphoma from carcinoma). Subtle and very small infiltrates can also be revealed by using immunohistochemistry, as demonstrated in this paper. Furthermore, immunohistochemistry shows that these infiltrates are more extensive than is apparent on routine stains. In some of the cases the nodular configuration of the infiltrate only became apparent on the immunostained section. Re-examination of the routinely stained bone marrow trephine specimen reported as negative for lymphoma, showed atypical lymphoid cells in two of five cases. These were intimately admixed with the normal cellular constituents of the marrow and a confident, unequivocal diagnosis could not be made. It must be said detection of these cells was made easier with the knowledge of the immunohistochemical findings. Confluent clusters were not easily discerned.

The role of anti-bcl-2 in distinguishing between benign and malignant infiltrates in the marrow is not clear cut. Staining of trephine specimens with anti-bcl-2 is of use in separating benign from malignant lymphoid cells. ${ }^{6}$ However, in follicular lymphomas within lymph nodes, bcl-2 positive bone marrow infiltrates are more likely to be malignant than benign.

Recent techniques such as the polymerase chain reaction (PCR) have been used to detect minimal residual disease. ${ }^{78}$ This provides a sensitive diagnostic method that can detect one 
to 10 malignant cells within a population of $10^{6}$ normal cells. However, to amplify the relevant cells, they must be detected by conventional light microscopy and subsequently microdissected to prevent contamination. The coupling of immunohistochemistry with PCR would, in this instance, be complimentary. Immunohistochemistry has the decided advantage of being technically simple, cheap and operable on a routine basis.

This paper emphasises the use of immunohistochemistry (even a limited panel of markers) in routinely fixed bone marrow trephine specimens and highlights the use of antiCD79a and anti-bcl-2 in the recognition of minimal bone marrow involvement by follicular lymphoma.
1 Van der Valk P, Mullink H, Huijgens PC, Tadema TM, Vos W, Meijer CJLM. Immunohistochemistry in bone marrow diagnosis. Am f Surg Pathol 1989;13:97-106.

2 Shin SS, Sheibani K, Kezirian J, Nademanee A, Forman SJ, Lee SK, et al. Immunoarchitecture of normal human bone marrow: A study of frozen and fixed tissue sections. Hum Pathol 1992;23:686-94.

3 Clark P, Normansell DE, Innes DJ, Hess CE. Lymphocyte subsets in normal bone marrow. Blood 1986;67:1600-6.

4 Rywlin AM, Ortega RS, Dominguez GJ. Lymphoid nodules of bone marrow, normal and abnormal. Blood 1974;13: of bone $389-400$.

5 Brunning RD, McKenna RW. Bone marrow manifestations of malignant lymphoma and lymphoma-like conditions Pathol Annu 1979;1:1-58.

6 Neilson JR, Oates JL, Leyland ML, Crocker J. Patterns of bcl-2 staining in bone marrow biopsies from patients with follicular lymphoma. F Pathol 1995;175:154A

7 Breit TM, Beishuizen A, Ludwig W-D, Mol EJ, Adriaansen $\mathrm{HJ}$, van Wering ER, et al. tal-1 deletions in T-cell acute lymphoblastic leukemia as PCR target for detection of minimal residual disease. Leukemia 1993;7:2004-11.

8 van Dongen JIM, Breit TM, Adriaansen HJ, Beishuizen A Hooijkas $H$. Detection of minimal residual disease in acute leukemia by immunologic marker analysis and polymerase chain reaction. Leukemia 1992;6:47-59. 